\title{
Two-step multi-spectral registration via key-point detector and gradient similarity. Application to agronomic scenes for proxy-sensing
}

\author{
Jehan-Antoine VAYSSADE ${ }^{1} \mathbb{C}^{\mathrm{a}}$, Gawain Jones ${ }^{1} \mathbb{C}^{\mathrm{b}}$, Jean-Noel Paoli ${ }^{1} \mathbb{C}^{\mathrm{c}}$ and Christelle Gee $^{1} \mathbb{C}^{\mathrm{d}}$ \\ ${ }^{1}$ Agroécologie, AgroSup Dijon, INRA, Univ. Bourgogne-Franche-Comté, F-21000 Dijon, France \\ jehan-antoine.vayssade@inra.fr, \{gawain.jones, jean-noel.paoli, christelle.gee\}@agrosupdijon.fr
}

\author{
Keywords: \\ Registration, Multi-spectral imagery, Precision farming, Feature descriptor
}

\begin{abstract}
:
The potential of multi-spectral images is growing rapidly in precision agriculture, and is currently based on the use of multi-sensor cameras. However, their development usually concerns aerial applications and their parameters are optimized for high altitudes acquisition by drone $(\mathrm{UAV} \approx 50$ meters) to ensure surface coverage and reduce technical problems. With the recent emergence of terrestrial robots (UGV), their use is diverted for nearby agronomic applications. Making it possible to explore new agronomic applications, maximizing specific traits extraction (spectral index, shape, texture ...) which requires high spatial resolution. The problem with these cameras is that all sensors are not aligned and the manufacturers' methods are not suitable for close-field acquisition, resulting in offsets between spectral images and degrading the quality of extractable informations. We therefore need a solution to accurately align images in such condition.

In this study we propose a two-steps method applied to the six-bands Airphen multi-sensor camera with (i) affine correction using pre-calibrated matrix at different heights, the closest transformation can be selected via internal GPS and (ii) perspective correction to refine the previous one, using key-points matching between enhanced gradients of each spectral bands. Nine types of key-point detection algorithms (ORB, GFTT, AGAST, FAST, AKAZE, KAZE, BRISK, SURF, MSER) with three different modalities of parameters were evaluated on their speed and performances, we also defined the best reference spectra on each of them. The results show that GFTT is the most suitable methods for key-point extraction using our enhanced gradients, and the best spectral reference was identified to be the band centered on $570 \mathrm{~nm}$ for this one. Without any treatment the initial error is about $62 \mathrm{px}$, with our method, the remaining residual error is less than $1 \mathrm{px}$, where the manufacturer's involves distortions and loss of information with an estimated residual error of approximately $12 \mathrm{px}$.
\end{abstract}

\section{INTRODUCTION}

Modern agriculture is changing towards a system that is less dependent on pesticides [Lechenet et al., 2014] (herbicides remain the most difficult pesticides to reduce) and digital tools are of great help in his matter. The development of imaging and image processing have made it possible to characterize an agricultural plot [Sankaran et al., 2015] (crop health status or soil

\footnotetext{
a (iD https://orcid.org/0000-0002-7418-8347

b (D) https://orcid.org/0000-0002-5492-9590

c(D) https://orcid.org/0000-0002-0499-9398

d(D) https://orcid.org/0000-0001-9744-5433
}

characteristics) using non-destructive agronomic indices [Jin et al., 2013] replacing traditional destructive and time-consuming methods. In recent years, the arrival of miniaturized multi-spectral and hyper-spectral cameras on Unmanned Aerial Vehicles (UAVs) has allowed spatio-temporal field monitoring. These vision systems have been developed for precise working conditions (flight height $50 \mathrm{~m}$ ). Although, very practical to use, they are also used for proxy-sensing applications. However, the algorithms offered by manufacturers to co-register multiple single-band images at different spectral range, are not optimal for low heights. It thus requires a specific close-field image registration. 
Image registration is the process of transforming different images of one scene into the same coordinate system. The spatial relationships between these images can be rigid (translations and rotations), affine (shears for example), homographic, or complex large deformation models (due to the difference of depth between ground and leafs) [Kamoun, 2019]. The main difficulty is that multi-spectral cameras have low spectral coverage between bands, resulting in a loss of characteristics between them. Which is caused by (i) plant leaves have different aspect depending on the spectral bands (ii) there are highly complex and self-similar structures in our images [Douarre et al., 2019]. It therefore affects the process of detecting common characteristics between bands for image registration. There are two types of registration, feature based and intensity based [Zitová and Flusser, 2003]. Feature based methods works by extracting point of interest and use feature matching, in most cases a brute-force matching is used, making those techniques slow. Fortunately these features can be filtered on the spatial properties to reduce the matching cost. A GPGPU implementation can also reduce the comparisons cost. Intensitybased automatic image registration is an iterative process, and the metrics used are sensitive to determine the numbers of iteration, making such method computationally expensive for precise registration. Furthermore multi-spectral implies different metrics for each registered bands which is hard to achieve.

Different studies of images alignment using multi-sensors camera can be found for acquisition using UAV at medium $(50-200 \mathrm{~m})$ and high $(200-1000 \mathrm{~m})$ distance. Some show good performances (in term of number of key-points) of feature based [Dantas Dias Junior et al., 2019, Vakalopoulou and Karantzalos, 2014] with strong enhancement of feature descriptor for matching performances. Other prefer to use intensity based registration [Douarre et al., 2019] on better convergence metrics [Chen et al., 2018] (in term of correlation), which is slower and not necessarily robust against light variability and their optimization can also fall into a local minimum, resulting in a non-optimal registration [Vioix, 2004].

Traditional approach to multi-spectral image registration is to designate one channel as the target channel and register all the others on the selected one. Currently, only [Dantas Dias Junior et al., 2019] show a method for selecting the best reference, but there is no study who as defined the best spectral reference in agronomic scene. In all cases NIR (850 nm) or middle range spectral reference are conventionally used without studying the others on precision agriculture. In addition those studies mainly propose features matching without large methods comparison [Dantas Dias Junior et al., 2019](less than 4) of their performance (time/precision), without showing the importance of the spectral reference and the interest of normalized gradients transformation (like in Intensity-based methods).

However, despite the growing use of UGVs and multi-spectal imaging, the domain is not very well sourced, and no study has been found under agricultural and external conditions in near field of view (less than 10 meter) for multi-spectral registration.

Thus, this study propose a benchmark of popular feature extractors inside normalized gradients transformation and the best spectral reference was defined for each of them. Moreover a pre-affine registration is used to filter the feature matching, evaluated at different spatial resolutions. So this study shows the importance of the selection of the reference and the features extractor on normalized gradients in such registration.

\section{MATERIAL AND METHOD}

\subsection{Material}

\subsubsection{Camera}

The multi-spectral imagery is provided by the six-band multi-spectral camera Airphen developed by HiPhen. Airphen is a scientific multi-spectral camera developed by agronomists for agricultural applications. It can be embedded in different types of platforms such as UAV, phenotyping robots, etc.

Airphen is highly configurable (bands, fields of view), lightweight and compact. The camera was configured using interferential filter centered at $450 / 570 / 675 / 710 / 730 / 850 \mathrm{~nm}$ with $\mathrm{FWHM}^{1}$

\footnotetext{
${ }^{1}$ Full Width at Half Maximum
} 
of $10 \mathrm{~nm}$, the position of each band is referenced on figure 1 . The focal lens is $8 \mathrm{~mm}$ for all wavelength. The raw resolution for each spectral band is $1280 \times 960$ px with 12 bit of precision. Finally the camera also provides an internal GPS antenna that can be used to get the distance from the ground.

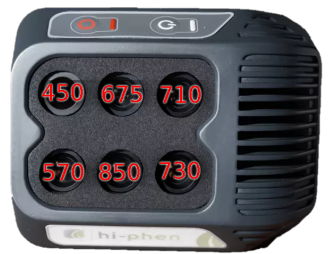

Figure 1: Disposition of each band on the Airphen multi-sensors camera

\subsubsection{Datasets}

Two datasets were taken at different heights with images of a chessboard (use for calibration) and of an agronomic scene. We used a metallic gantry for positioning the camera at different heights. The size of the gantry is $3 \times 5 \times 4 \mathrm{~m}$. Due to the size of the chessboard $(57 \times 57 \mathrm{~cm}$ with $14 \times 14$ square of $4 \mathrm{~cm}$ ), the limited focus of the camera and the gantry height, we have bounded the acquisition heights from 1.6 to $5 \mathrm{~m}$ with $20 \mathrm{~cm}$ steps, which represents 18 acquisitions.

The first dataset is for the calibration. A chessboard is taken at different heights The second one is for the alignment verification under real conditions. One shot of an agronomic scene is taken at different heights with a maximum bias set at $10 \mathrm{~cm}$.

\subsection{Methods}

Alignment is refined in two stages, with (i) affine registration approximately estimated and (ii) perspective registration for the refinement and precision. As example the figure 2 shows each correction step, where the first line is for the (i) affine correction (section 2.2.1), the second is for (ii) perspective correction. More precisely the second step is per-channel pre-processed where feature detectors are used to detect key-points (section 2.2.2). Each channel key-points are associated to compute the perspective correction through homography, to the chosen spectral band (section 2.2.2). These steps are explained on specific subsections.

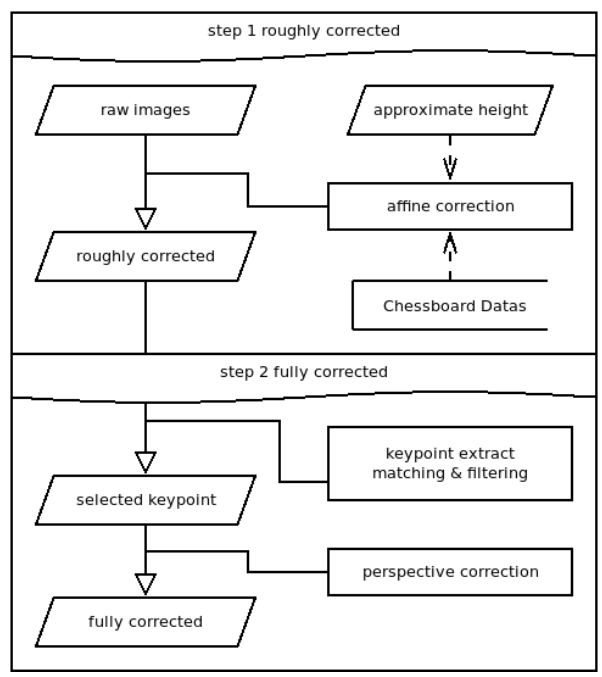

Figure 2: Each step of the alignment procedure, with (step 1) roughly corrected from affine correction and (step 2) enhancement via key-points and perspective

\subsubsection{Affine Correction}

We make the assumption that closer we take the snapshot, the bigger the distance between each spectral band is. On the other hand, at a distance superior or equals to $5 \mathrm{~m}$, the initial affine correction become stable. A calibration is used to build a linear model based on that assumption, which will allow the affine correction to work at any height. The main purpose of this step is to reduce the offset between each spectral band, which allows the similarity between key-points to be spatially delimited within a few pixels, making feature matching more effective.

Calibration : Based on that previous assumption a calibration is run over the chessboard dataset. We detect the chessboard using the opencv calibration toolbox [Bouguet, 2001] on each spectral image (normalized by $I=(I-$ $\min (I)) / \max (I)$ where $I$ is the spectral image) at different heights (from $1.6 \mathrm{~m}$ to $5 \mathrm{~m}$ ). We use the function findChessboardCorners how attempts to determine whether the input image is a view of the chessboard pattern and locate the internal chessboard corners. The detected coordinates are roughly approximated. To determine their positions accurately we use the function cornerSubPix as explained in the documentation ${ }^{2}$.

\footnotetext{
2 https://docs.opencv.org/2.4/modules/calib3d/ doc/camera_calibration_and_3d_reconstruction. html
} 
Linear model : Using all the points detected for each spectral band, we calculate the centroid grid (each point average). The affine transform from each spectral band to this centroid grid is estimated. Theoretically, the rotation and the scale $(A, B, C, D)$ do not depend on the distance to the ground, but the translation $(X, Y)$ does. Thus a Levenberg-Marquardt curve fitting algorithm with linear least squares regression [Moré, 1978] can be used to fit an equation for each spectral band against $X$ and $Y$ independently to the centroid grid. We adjust the following curve $t=\alpha h^{3}+\beta h^{2}+\theta h+\gamma$ where $h$ is the height, $t$ is the resulted translation and factors $\alpha, \beta, \theta, \gamma$ are the model parameters.

Correction : Based on the model estimated on the chessboard dataset, we transpose them to the agronomic dataset. To make the affine matrix correction, we used the rotation and scale factors at the most accurate height $(1.6 \mathrm{~m}$ where the spatial resolution of the chessboard is higher), because it does not theoretically depend on the height. For the translation part, the curve model is applied for each spectral band at the given height provided by the user. Each spectral band is warped using the corresponding affine transformation. Finally, all spectral bands are cropped to the minimal bounding box (minimal and maximal translation of each affine matrix). This first correction is an approximation. It provides some spatial properties that we will use on the second stage.

\subsubsection{Perspective correction}

Each spectral band has different properties and values by nature but we can extract the corresponding similarity by transforming each spectral band into its absolute derivative, to find similarities in gradient break among them. As we can see in figure 3 gradients can have opposite direction depending on the spectral bands, making the absolute derivative an important step for matching between different spectral band.
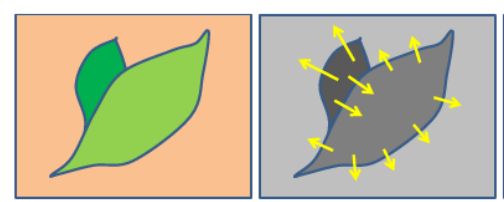

Visible

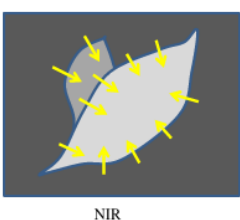

NIR
Figure 3: Gradient orientation in spectral band [Rabatel and Labbe, 2016]. Orientation of the gradient is not the same depending to the spectral band.
The affine correction attempts to help the feature matching by adding properties of epipolar lines (close). Thus, the matching of extracted features can be spatially bounded, (i) we know that the maximum translation is limited to a distance of a few pixels (less than 10px thanks to affine correction), and (ii) the angle between the initial and the matched one is limited to $[-1,1]$ degree.

Computing the gradient : To compute the gradient of the image with a minimal impact of the light distribution (shadow, reflectance, specular, ...), each spectral band is normalized using Gaussian blur [Sage and Unser, 2003], the kernel size is defined by next_odd(image_widt $\left.h^{0.4}\right)$ (19 in our case) and the final normalized images are defined by $I /(G+1) * 255$ where $I$ is the spectral band and $G$ is the Gaussian blur of those spectral bands. This first step minimizes the impact of the noise on the gradient and smooth the signal in case of high reflectance. Using this normalized image, the gradient $I_{\text {grad }}(x, y)$ is computed with the sum of absolute Sharr filter [Seitz, 2010] for horizontal $S_{x}$ and vertical $S_{y}$ derivative, noted $I_{\text {grad }}(x, y)=\frac{1}{2}\left|S_{x}\right|+\frac{1}{2}\left|S_{y}\right|$. Finally, all gradients $I_{\text {grad }}(x, y)$ are normalized using CLAHE [Zuiderveld, 1994] to locally improve their intensity and increase the number of keypoints detected.

Key-points Extractor : A key-point is a point of interest. It defines what is important and distinctive in an image. Different types of key-point extractors are available and the following are tested :

(ORB) Oriented FAST and Rotated BRIEF [Rublee et al., 2011], (AKAZE) Fast explicit diffusion for accelerated features in nonlinear scale spaces [Alcantarilla and Solutions, 2011], (KAZE) A novel multi-scale 2D feature detection and description algorithm in nonlinear scale spaces [Ordonez et al., 2018], (BRISK) Binary robust invariant scalable key-points [Leutenegger et al., 2011], (AGAST) Adaptive and generic corner detection based on the accelerated segment test [Mair et al., 2010], (MSER) maximally stable extremal regions [Donoser and Bischof, 2006], (SURF) Speed-Up Robust Features [Bay et al., 2006], (FAST) FAST Algorithm for Corner Detection [Trajković and Hedley, 1998] and (GFTT) Good Features To Track [Shi et al., 1994]. 
These algorithms are largely described across multiple studies [Dantas Dias Junior et al., 2019, Tareen and Saleem, 2018, Zhang et al., 2016, Ali et al., 2016], they are all available and easily usable in OpenCV. Thus we have studied them by varying the most influential parameters for each of them with three modalities, the table 1 in appendix shows all modalities.

Table 1: list of algorithms with 3 modalities of their parameters

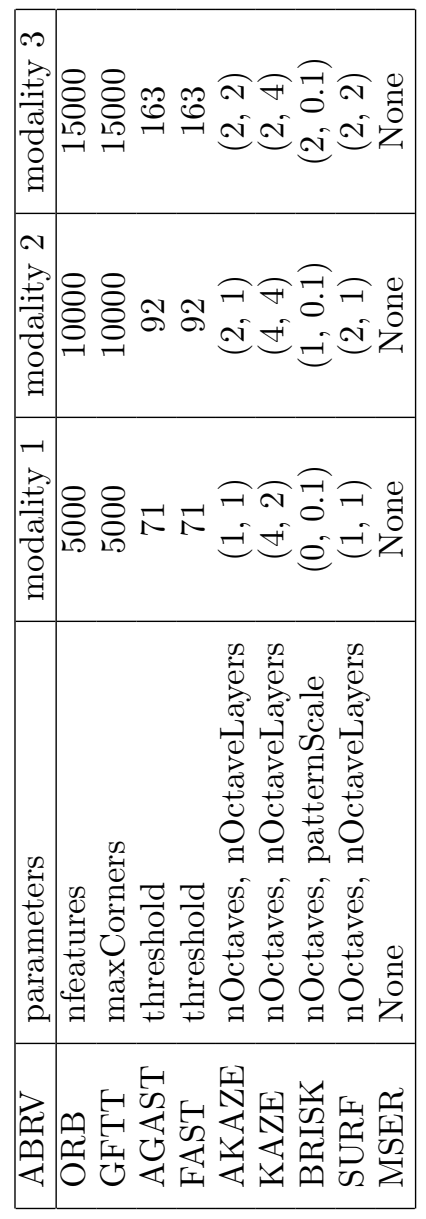

Key-point detection : We use one of the keypoint extractors mentioned above between each spectral band gradients (all extractors are evaluated). For each detected key-point, we extract a descriptor using ORB features. We match all detected key-points to a reference spectral band (all bands are evaluated). All matches are filtered by distance, position and angle, to eliminate a majority of false positives along the epipolar line. Finally we use the function findHomography between the key-points detected/filtered with RANSAC [Fischler and Bolles, 1981], to determine the best subset of matches to calculate the perspective correction.

Correction : The perspective correction between each spectral band to the reference is estimated and applied. Finally, all spectral bands are cropped to the minimum bounding box, which is obtained by applying a perspective transformation to each corner of the image.

\section{RESULTS AND DISCUSSION}

Firstly the results will focus on affine corrections and then on the effects of the perspective correction. Figure 4 shows a closeup inside at $1.6 \mathrm{~m}$ (4a) raw images acquisition, (4c \& 4d) registred image of each correction steps and (4b) the manufacturer results.

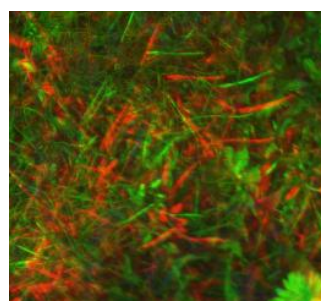

(a) raw image

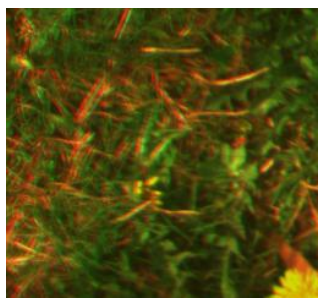

(c) roughly corrected

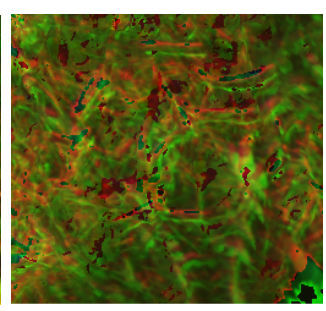

(b) manufacturer's

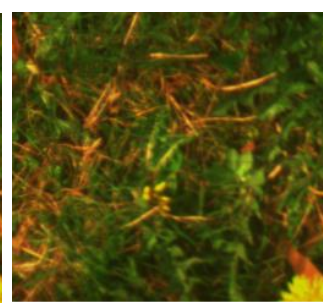

(d) fully corrected
Figure 4: Example of each correction and the manufacturers results

\subsection{Affine correction}

The affine correction model is based on the calibration dataset (where the chessboard are acquired). The 6 coefficients $(A, B, C, D, X, Y)$ of the affine matrix were studied according to the height of the camera in order to see their stability. It appears that the translation part $(X, Y)$, depends on the distance to the field (appendix figure 5) according to the initial assumption. On this part the linear model is used to estimate the affine correction from an approximated height. 

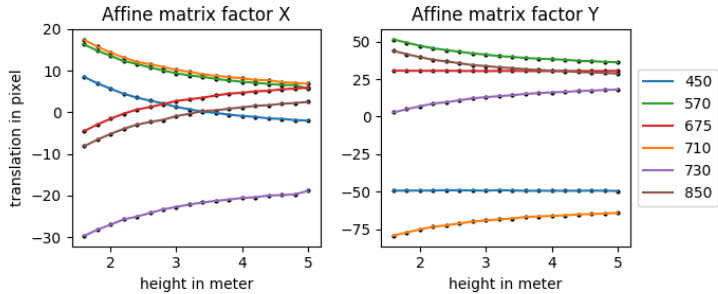

Figure 5: Affine matrix value by height

Rotation and scale do not depend on the ground distance (figure 6) according to the theory. These factors $(A, B, C, D)$ are quite stable and close to identity, as expected (accuracy depends on the spatial resolution of the board). As result, single calibration can be used for this part of the matrix, and the most accurate are used (i.e where the chessboard has the best spatial resolution).
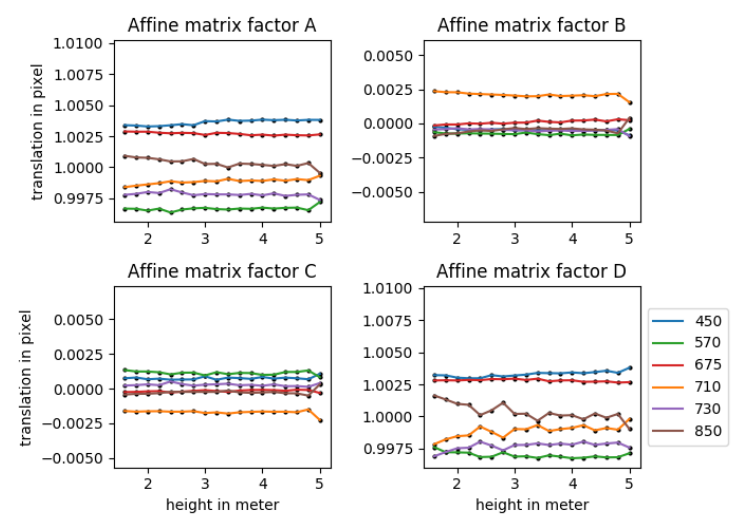

Figure 6: Affine matrix value by height

After the affine correction, the remaining residual distances have been extracted, it is computed using the detected, filtered and matched key-point to the reference spectral band, figure 9 (up) shows an example using $570 \mathrm{~nm}$ as reference before the perspective correction. The remaining distance between each spectral band to the reference varies according to the distance between the real height and the nearest selected (through linear model). Remember that a bias of $+/-10 \mathrm{~cm}$ was initially set to show the error in the worst case, so the difference of errors between each of them are due to the difference of sensors position in the array to the reference and the provided approximate height.

\subsection{Perspective correction}

The figures 7 shows the numbers of key-points after filtering and homographic association (min- imum of all matches) as well as the computation time and performance ratio (matches/time) for each method. The performance ratio is used to compare methods between them, bigger he is, greater is the method (balanced between time and accuracy), making lower of them unsuitable.

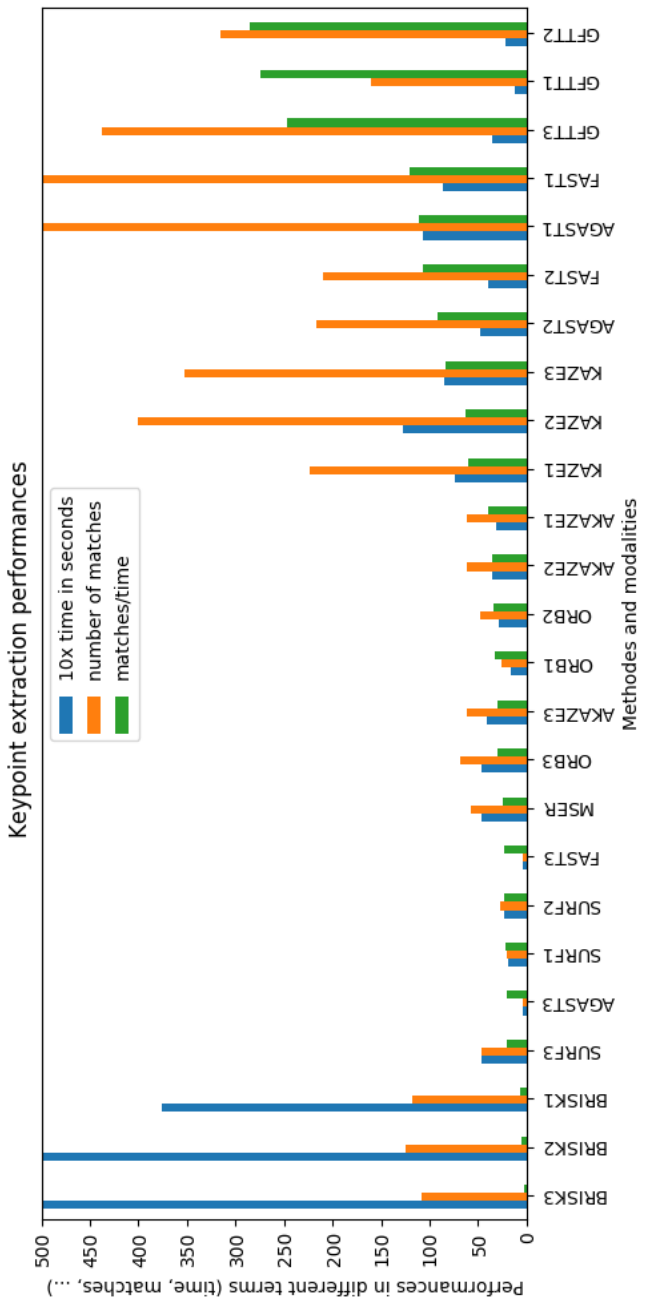

Figure 7: features extractor performances after filtering and homography association

All these methods offer interesting results, the choice of method depends on application needs between computation time and accuracy, three methods stand out in all of there modality:

- GFTT shows the best overall performance in both computation time and number of matches

- FAST and AGAST1 are quite suitable too, with acceptable computation time and greater matches performances. 
The other ones did not show improvement in term of time or matches (especially compared to GFTT), some of them show a small number of matches which can be too small to ensure the precision. Increasing the number of keypoints matched allows a slightly higher accuracy [Dantas Dias Junior et al., 2019]. For example, switching from SURF (30 results) to FAST (130 results) reduces the final residual distances from $\approx 1.2$ to $\approx 0.9 \mathrm{px}$ but increases the calculation time from $\approx 5$ to $\approx 8$ seconds.

All methods show that the best spectral band is $710 \mathrm{~nm}$ (in red), with an exception for SURF and GFTT which is $570 \mathrm{~nm}$. The figure 8 shows the minimum number of matches between each reference spectrum and all the others, for each relevant methods and modalities (KAZE, AGAST, FAST GFTT). Choosing the right spectral reference is important, as we can see, no correspondence is found in some cases between $675-850 \mathrm{~nm}$, but correspondences are found between 675-710 $\mathrm{nm}$ and 710-850 $\mathrm{nm}$, making the $710 \mathrm{~nm}$ more appropriate, the same behavior can be observed for the other bands and $570 \mathrm{~nm}$ as the more appropriate one. This is visible on the figure for all methods, $570 \mathrm{~nm}$ and $710 \mathrm{~nm}$ have the best minimum number of matches where all the other are quite small.

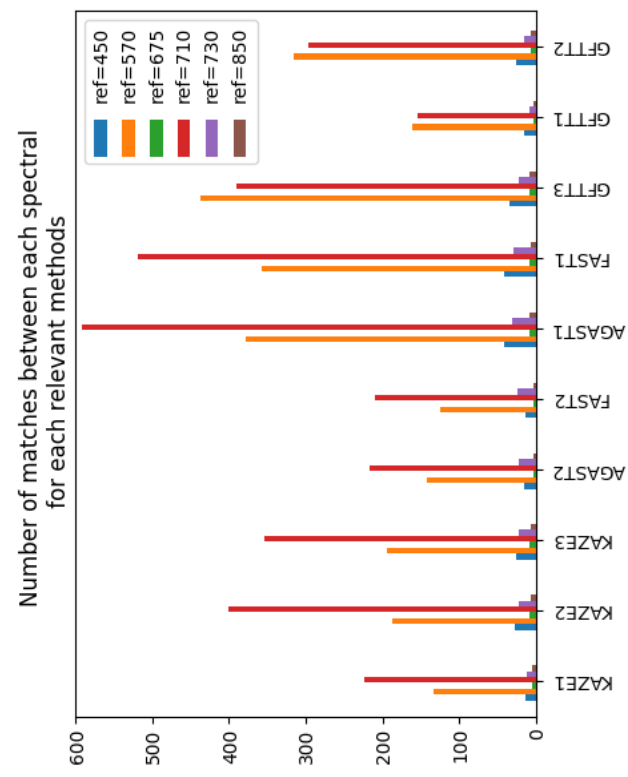

Figure 8: key-point extractor performances

Residues of the perspective correction show that we have correctly registered each spectral band, the figure 9 (down) shows the residual distance at different ground distances. In comparison the affine correction error are between $[1.0-4.8]$ px where the combination of affine and perspective correction the residual error are between $[0.7-1.0]$ px. On average the perspective correction enhance the residual error by $(3.5-0.9) / 3.5 \approx 74 \%$.
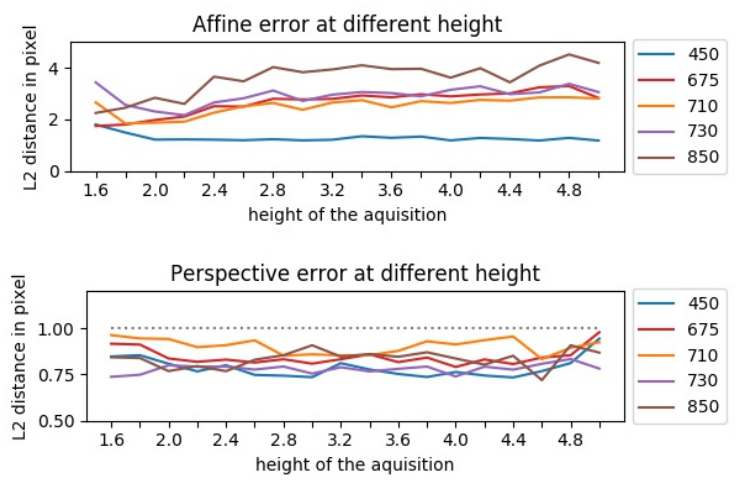

Figure 9: (up) The mean distance of detected keypoint before perspective correction with $570 \mathrm{~nm}$ as spectral reference (down) Perspective re-projection error with GFTT using the first modality and 570 $\mathrm{nm}$ as reference

\subsection{General discussion}

Even if the relief of the scene is not taken into account due to the used deformation model, in our case, with flat ground, no difference arise. However, more complex deformation models [Lombaert et al., 2012, Bookstein, 1989] could be used to improve the remaining error. But could also, in some case, create large angular deformations caused by the proximity of key-points, of course, it's possible to filter these key-points, which would also reduce the overall accuracy.

Further research can be performed on each parameter of the feature extractors, for those who need specific performance (time/precision), we invite anyone to download the dataset and test various combinations. Otherwise feature matching can be optimized, at this stage, we use bruteforce matching with post filtering, but a different implementation that fulfill our spatial properties should greatly enhance the number of matches by reducing false positives. 


\section{CONCLUSION}

In this work, the application of different techniques for multi-spectral image registration was explored using the Airphen camera. We have tested nine type of key-points extractor (ORB, GFTT, AGAST, FAST, AKAZE, KAZE, BRISK, SURF, MSER) at different heights and the number of control points obtained. As seen in the method, the most suitable method is the GFTT (regardless of modalities 1,2 or 3) with a significant number of matches $150-450$ and a reasonable calculation time $1.17 \mathrm{~s}$ to $3.55 \mathrm{~s}$ depending on the modality.

Furthermore, the best spectral reference was defined for each method, for example $570 \mathrm{~nm}$ for GFTT. We have observed a residual error of less than $1 \mathrm{px}$, supposedly caused by the difference of sensors nature (spectral range, lens).

\section{ACKNOWLEDGMENTS}

The authors acknowledge support from European Union through the project H2020 IWMPRAISE $^{3}$ (Integrated Weed Management: PRActical Implementation and Solutions for Europe) and from ANR Challenge ROSE through the project ROSEAU ${ }^{4}$ (RObotics SEnsorimotor loops to weed AUtonomously).

We would like to thanks Combaluzier Quentin, Michon Nicolas, Savi Romain and Masson JeanBenoit for the realization of the metallic gantry that help us positioning the camera at different heights.

\section{REFERENCES}

[Alcantarilla and Solutions, 2011] Alcantarilla, P. F. and Solutions, T. (2011). Fast explicit diffusion for accelerated features in nonlinear scale spaces. IEEE Trans. Patt. Anal. Mach. Intell, 34(7):12811298.

[Ali et al., 2016] Ali, F., Khan, S. U., Mahmudi, M. Z., and Ullah, R. (2016). A comparison of fast, surf, eigen, harris, and mser features. International Journal of Computer Engineering and Information Technology, 8(6):100.

\footnotetext{
${ }^{3}$ https://iwmpraise.eu/

${ }^{4}$ http://challenge-rose.fr/en/projet/roseau-2/
}

[Bannari et al., 1995] Bannari, A., Morin, D., Bonn, F., and Huete, A. R. (1995). A review of vegetation indices. Remote Sensing Reviews, 13(1-2):95-120.

[Bay et al., 2006] Bay, H., Tuytelaars, T., and Van Gool, L. (2006). Surf: Speeded up robust features. In European conference on computer vision, pages 404-417. Springer.

[Bookstein, 1989] Bookstein, F. L. (1989). Principal warps: thin-plate splines and the decomposition of deformations. IEEE Transactions on Pattern Analysis and Machine Intelligence, 11(6):567-585.

[Bouguet, 2001] Bouguet, J.-Y. (2001). Camera calibration toolbox for matlab.

[Chen et al., 2018] Chen, S., Shen, H., Li, C., and Xin, J. H. (2018). Normalized total gradient: A new measure for multispectral image registration. IEEE Transactions on Image Processing, 27(3):1297-1310.

[Dantas Dias Junior et al., 2019] Dantas Dias Junior, J., Backes, A., and Escarpinati, M. (2019). Detection of control points for uav-multispectral sensed data registration through the combining of feature descriptors. pages 444-451.

[Donoser and Bischof, 2006] Donoser, M. and Bischof, H. (2006). Efficient maximally stable extremal region (mser) tracking. In 2006 IEEE Computer Society Conference on Computer Vision and Pattern Recognition (CVPR'06), volume 1, pages 553-560. Ieee.

[Douarre et al., 2019] Douarre, C., Crispim-Junior, C. F., Gelibert, A., Tougne, L., and Rousseau, D. (2019). A strategy for multimodal canopy images registration. In 7th International Workshop on Image Analysis Methods in the Plant Sciences, Lyon, France.

[Filella et al., 1995] Filella, I., Serrano, L., Serra, J., and Penuelas, J. (1995). Evaluating wheat nitrogen status with canopy reflectance indices and discriminant analysis. Crop Science, 35(5):1400-1405.

[Fischler and Bolles, 1981] Fischler, M. A. and Bolles, R. C. (1981). Random sample consensus: A paradigm for model fitting with applications to image analysis and automated cartography. Commun. ACM, 24(6):381-395.

[Jin et al., 2013] Jin, X.-l., Diao, W.-y., Xiao, C.-h., Wang, F.-y., Chen, B., Wang, K.-r., and Li, S.-k. (2013). Estimation of wheat agronomic parameters using new spectral indices. PLOS ONE, 8(8):1-9.

[Kamoun, 2019] Kamoun, E. (2019). Image registration: From sift to deep learning.

[Lechenet et al., 2014] Lechenet, M., Bretagnolle, V., Bockstaller, C., Boissinot, F., Petit, M.-S., Petit, S., and Munier-Jolain, N. M. (2014). Reconciling pesticide reduction with economic and environmental sustainability in arable farming. PLOS ONE, 9(6):1-10. 
[Leutenegger et al., 2011] Leutenegger, S., Chli, M., and Siegwart, R. (2011). Brisk: Binary robust invariant scalable keypoints. In 2011 IEEE international conference on computer vision (ICCV), pages $2548-2555$. Ieee.

[Lombaert et al., 2012] Lombaert, H., Grady, L., Pennec, X., Ayache, N., and Cheriet, F. (2012). Spectral demons - image registration via global spectral correspondence. In Fitzgibbon, A., Lazebnik, S., Perona, P., Sato, Y., and Schmid, C., editors, Computer Vision - ECCV 2012, pages 30-44, Berlin, Heidelberg. Springer Berlin Heidelberg.

[Mair et al., 2010] Mair, E., Hager, G. D., Burschka, D., Suppa, M., and Hirzinger, G. (2010). Adaptive and generic corner detection based on the accelerated segment test. In European conference on Computer vision, pages 183-196. Springer.

[Moré, 1978] Moré, J. J. (1978). The levenbergmarquardt algorithm: Implementation and theory. In Watson, G., editor, Numerical Analysis, volume 630 of Lecture Notes in Mathematics, pages 105116. Springer Berlin Heidelberg.

[Ordonez et al., 2018] Ordonez, A., Arguello, F., and Heras, D. B. (2018). Alignment of hyperspectral images using kaze features. Remote Sensing, 10(5).

[Rabatel and Labbe, 2016] Rabatel, G. and Labbe, S. (2016). Registration of visible and near infrared unmanned aerialvehicle images based on Fourier-Mellin transform. Precision Agriculture, 17(5):564-587.

[Rublee et al., 2011] Rublee, E., Rabaud, V., Konolige, K., and Bradski, G. (2011). Orb: An efficient alternative to sift or surf. In Proceedings of the 2011 International Conference on Computer Vision, ICCV '11, pages 2564-2571, Washington, DC, USA. IEEE Computer Society.

[Sage and Unser, 2003] Sage, D. and Unser, M. (2003). Teaching image-processing programming in java. IEEE Signal Processing Magazine, 20(6):4352. Using "Student-Friendly" ImageJ as a Pedagogical Tool.

[Sankaran et al., 2015] Sankaran, S., Khot, L. R., Espinoza, C. Z., Jarolmasjed, S., Sathuvalli, V. R., Vandemark, G. J., Miklas, P. N., Carter, A. H., Pumphrey, M. O., Knowles, N. R., and Pavek, M. J. (2015). Low-altitude, high-resolution aerial imaging systems for row and field crop phenotyping: A review. European Journal of Agronomy, 70:112 - 123 .

[Seitz, 2010] Seitz, H. (2010). Contributions to the minimum linear arrangement problem.

[Shi et al., 1994] Shi, J. et al. (1994). Good features to track. In 1994 Proceedings of IEEE conference on computer vision and pattern recognition, pages 593-600. IEEE.

[Tareen and Saleem, 2018] Tareen, S. A. K. and Saleem, Z. (2018). A comparative analysis of sift, surf, kaze, akaze, orb, and brisk. 2018 International
Conference on Computing, Mathematics and Engineering Technologies (iCoMET), pages 1-10.

[Trajković and Hedley, 1998] Trajković, M. and Hedley, M. (1998). Fast corner detection. Image and vision computing, 16(2):75-87.

[Vakalopoulou and Karantzalos, 2014] Vakalopoulou, M. and Karantzalos, K. (2014). Automatic descriptor-based co-registration of frame hyperspectral data. Remote Sensing, 6 .

[Vioix, 2004] Vioix, J.-B. (2004). Conception et réalisation d'un dispositif d'imagerie multispectrale embarqué : du capteur aux traitements pour la détection d'adventices.

[Zhang et al., 2016] Zhang, H., Wohlfeil, J., and Grießbach, D. (2016). Extension and evaluation of the agast feature detector.

[Zitová and Flusser, 2003] Zitová, B. and Flusser, J. (2003). Image registration methods: A survey. Image and Vision Computing, 21:977-1000.

[Zuiderveld, 1994] Zuiderveld, K. (1994). Contrast limited adaptive histogram equalization. In Graphics gems IV, pages 474-485. Academic Press Professional, Inc. 